\title{
Short-bunch generation from an rf photoinjector with injection phase compensation
}

\author{
F. H. Chao, ${ }^{1}$ C. H. Chen, ${ }^{2,3}$ Y. C. Huang, ${ }^{1,2, *}$ and P. J. Chou ${ }^{1,3}$ \\ ${ }^{1}$ Department of Physics, National Tsing Hua University, Hsinchu 30013, Taiwan \\ ${ }^{2}$ Department of Electrical Engineering/Institute of Photonics, National Tsing Hua University, \\ Hsinchu 30013, Taiwan \\ ${ }^{3}$ National Synchrotron Radiation Research Center, Hsinchu 30076, Taiwan
}

(Received 25 November 2014; published 7 May 2015)

\begin{abstract}
A radio-frequency photoinjector is widely used for generating picosecond-femtosecond electron bunches. During particle acceleration, the electron bunch shape varies under the influence of both the acceleration fields and space charge fields. To restore a distorted electron bunch, we propose an injection phase compensation technique for the electrons by illuminating the photocathode with a pulse-front-corrected laser pulse. Using an S-band 1.6-cell photoinjector as an example, our study shows that the proposed technique can generate nearly undistorted electron bunches up to a bunch rate of a few hundred terahertz.
\end{abstract}

DOI: 10.1103/PhysRevSTAB.18.051301

PACS numbers: 07.77.Ka, 29.27.Ac, 41.60.Cr, 41.75.Jv

\section{INTRODUCTION}

Ultrashort electron bunches are becoming available from advanced accelerator technologies and finding a variety of applications. For example, electron bunching is crucial for radiation generation. When the electron bunch length is significantly shorter than the radiation wavelength, all the electrons in the bunch can radiate coherently. An electronpulse train can further generate radiation with a narrow spectrum at the pulse frequency or at the harmonics of the pulse frequency due to the constructive interference of the fields radiated from periodic electron bunches. In a freeelectron laser (FEL), a prebunched electron beam with periodic current modulation can generate copious harmonic power upon entering an undulator and is advantageous in rapidly building up the FEL power in a short undulator [1-6]. Another important application of a short electron bunch is ultrafast particle imaging that utilizes an ultrashort electron bunch to form a diffraction image with high temporal resolution [7-13]. To study lattice vibrations, phase transitions, chemical reactions, and strongly correlated electronic materials, a time-resolved measurement with subpicosecond resolution is preferred [7-13]. Recently, rf photoinjectors have been widely used as $\mathrm{MeV}$ electron sources in the applications of ultrafast electron diffraction (UED) imaging. For example, SLAC [9], University of California at Los Angeles (UCLA) [10], Tsinghua University at Beijing [7,8], and Osaka University [11-13] all established such UED facilities. In addition, an ultrafast

\footnotetext{
*ychuang@ee.nthu.edu.tw
}

Published by the American Physical Society under the terms of the Creative Commons Attribution 3.0 License. Further distribution of this work must maintain attribution to the author $(s)$ and the published article's title, journal citation, and DOI. electron microscopy (UEM) based on a photoinjector is under development at Osaka University [13].

In the past, it has been proposed to generate a terahertz electron-pulse train by illuminating a photocathode of an $\mathrm{rf}$ electron gun by a terahertz pulse-train laser [2-6]. To have appreciable electron bunching in an electron-pulse train, the longitudinal length of the electron microbunch should be significantly smaller than the inverse of the electronpulse rate. The final bunch length of an accelerated electron bunch can be sensitively influenced by the injection phase, initial bunch radius, space charge force, and acceleration gradient in an $\mathrm{rf}$ accelerator.

In this paper, we study the fundamental mechanisms of bunch lengthening and distortion in an rf photoinjector assuming delta-function-like electron bunches generated at the photocathode. In the subsequent discussions, we first define the bunching factor of an electron-pulse train and point out the dependence of the bunching factor on the length of the microbunch. In the absence of space charge force, we analyze the debunching behavior of a single delta-function electron bunch under the influence of radially dependent rf fields in an accelerator. Results of our theoretical model are compared with those simulated by the particle tracking code PARMELA [14]. To ameliorate the bunch lengthening and distortion, we propose to illuminate the photocathode with a pulse-front-shaped laser such that a shaped electron bunch [15-19] is generated and the bunch distortion during acceleration is corrected. It will be shown that the pulse-front-shaping technique is very effective in correcting the bunch distortion of both single- and multibunch electron beams during acceleration.

\section{BUNCH LENGTH AND BUNCHING FACTOR}

In radiation applications, the longitudinal length of an electron bunch is often compared with the radiation 
wavelength. To facilitate the discussions below, it is helpful to present here the quantitative description of an electron bunch and an electron bunch train. For a pulsed electron beam with a smooth temporal distribution described by $n(t)$, the so-called bunching amplitude or bunching factor [1-5] of the electron beam at a given radiation frequency $f$ is defined as

$B_{f}(f)=\frac{\left|\int_{-\infty}^{\infty} n(t) \exp (i 2 \pi f t) d t\right|}{\int_{-\infty}^{\infty} n(t) d t}=\frac{|\mathbb{F}\{n(t)\}|}{[\mathbb{F}\{n(t)\}]_{f=0}}$,

where $i$ is the imaginary unit and $\mathbb{F}\{n(t)\} \equiv \int_{-\infty}^{\infty} n(t)$ $\exp (i 2 \pi f t) d t$ is the Fourier transform of $n(t)$. Equation (1) indicates that $B_{f}(f)$ is highly dependent on the longitudinal distribution of electrons.

Suppose an electron macrobunch is composed of a periodic array of microbunches with a bunching frequency $f_{b}$ along the propagation direction. Further assume that each electron microbunch has a Gaussian-like particle distribution with a longitudinal root-mean-square (rms) bunch length of $\sigma_{\mu} . n(t)$, which describes the longitudinal distribution of such an electron-pulse train, can be expressed as

$$
n(t)=n_{0} \exp \left(-t^{2} / 2 \sigma_{\mu}^{2}\right) \otimes \operatorname{comb}\left(f_{b} t\right),
$$

where $A \otimes B$ is the convolution of $A$ and $B$ and $\operatorname{comb}\left(f_{b} t\right) \equiv \sum_{m=-\infty}^{\infty} \delta\left(f_{b} t-m\right)$ is a periodic array of the Dirac delta function in $t$. From Eqs. (1) and (2), the bunching factor of such an electron-pulse train is given by

$$
\begin{aligned}
B_{f}(f) & =\frac{\left|\mathbb{E}\left\{n_{0} \exp \left(-t^{2} / 2 \sigma_{\mu}^{2}\right) \otimes \operatorname{comb}\left(f_{b} t\right)\right\}\right|}{\left[\mathbb{E}\left\{n_{0} \exp \left(-t^{2} / 2 \sigma_{\mu}^{2}\right) \otimes \operatorname{comb}\left(f_{b} t\right)\right\}\right]_{f=0}} \\
& =\exp \left(-2 \sigma_{\mu}^{2} \pi^{2} f^{2}\right) \operatorname{comb}\left(f / f_{b}\right) \\
& =G(f) \operatorname{comb}\left(f / f_{b}\right) .
\end{aligned}
$$

According to Eq. (3), the bunching factor of the electronpulse train has a comblike spectrum in a Gaussian envelope $G(f) \equiv \exp \left(-2 \sigma_{\mu}^{2} \pi^{2} f^{2}\right)$, as shown by the dashed lines in Fig. 1(a). The blue, red, and black lines correspond to the bunching factors and Gaussian envelopes of Gaussian electron-pulse trains with rms microbunch lengths of $\sigma_{\mu}=0.1 / f_{b}, 0.2 / f_{b}$, and $0.3 / f_{b}$, respectively. When the microbunch length $\sigma_{\mu}$ increases, the bunching factor, denoted by the vertical lines, decays quickly in the Gaussian envelope. Figure 1(b) shows the relationship between $B_{f}\left(f=f_{b}\right)$ and the normalized bunch length $\sigma_{\mu} f_{b}$. From Figs. 1(a) and 1(b), we can see that the bunching factor of an electron-pulse train is sensitive to the microbunch length $\sigma_{\mu}$. For a bunching factor larger than $1 \%$, the rms microbunch length $\sigma_{\mu}$ has to be slightly less than half of the bunch rate period or $0.48 / f_{b}$, as Fig. 1(b) shows.

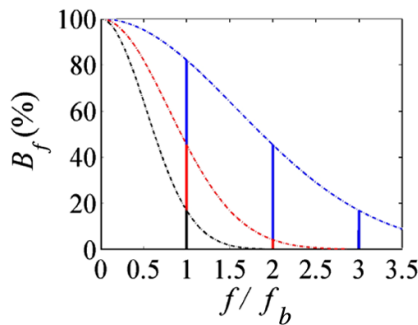

(a)

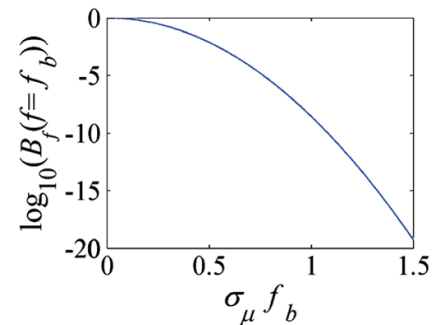

(b)
FIG. 1. (a) Bunching factor spectra $\left[B_{f}(f)\right.$, solid line] and Gaussian envelopes $[G(f)$, dashed line] of Gaussian electronpulse trains with rms microbunch lengths of $0.1 / f_{b}$ (blue curve), $0.2 / f_{b}$ (red curve), and $0.3 / f_{b}$ (black curve). (b) Bunching factor $B_{f}$ at $f=f_{b}$ versus normalized rms bunch length $\sigma_{\mu} f_{b}$ for a Gaussian electron-pulse train.

During the process of the emission and amplification of the radiation in the undulators, the power radiated from an electron beam at an arbitrary frequency $f$ is proportional to both the square of the beam current and the square of the bunching factor [1-5]. In other words, the radiation power can be enhanced by increasing the beam current or the bunching factor. In a superradiant FEL [1-3], it has been proposed to send an electron-pulse train into the undulator to enhance the growth of the radiation power and reduce the length of the undulator. However, the bunching factor of an electron-pulse train is usually deteriorated after electrons passing through accelerator components between the photoinjector and the undulator and becomes much smaller at the entrance of the undulator. Hence, it is important to compensate the bunch deformation and increase the bunching factor of the electron-pulse train generated from the photoinjector. In a photoinjector, the initial electron microbunch length is approximately the length of the incident driver laser pulse. However, during acceleration, the electron microbunch length and shape can be strongly influenced by the injection phase, acceleration fields, space charge force, and transverse distribution of electrons.

\section{BEAM DYNAMICS OF ULTRASHORT BUNCHES IN AN rf INJECTOR}

\section{A. Radially dependent rf fields}

In a photoinjector driven by an ultrafast laser, the electron bunches emitted from the cathode are typically accelerated by a standing wave. In an axially symmetrical, multicell, standing-wave rf accelerator, the dominant $\pi$-mode fields [20] are given by

$$
\begin{aligned}
E_{z}(r, z, t) & \approx E_{0} J_{0}\left(\eta_{0} r\right) \cos \left(k_{0} z\right) \sin \left(\omega t+\phi_{0}\right) \\
& =\frac{E_{0} J_{0}\left(\eta_{0} r\right)}{2}\left[\sin (\phi)+\sin \left(\phi+2 k_{0} z\right)\right], \\
E_{r}(r, z, t) & \approx \frac{k_{0}}{2 \eta_{0}} E_{0} J_{1}\left(\eta_{0} r\right)\left[\cos (\phi)-\cos \left(\phi+2 k_{0} z\right)\right],
\end{aligned}
$$


$B_{\theta}(r, z, t) \approx \frac{\omega}{2 c^{2} \eta_{0}} E_{0} J_{1}\left(\eta_{0} r\right)\left[\cos (\phi)+\cos \left(\phi+2 k_{0} z\right)\right]$,

where $k_{0}=\pi / L$ with $L$ being the cell length of the rf cavity, $\eta_{0}{ }^{2}=(\omega / c)^{2}-k_{0}^{2}, \phi=\omega t-k_{0} z+\phi_{0}$ is the rf phase [21], and $J_{0}$ and $J_{1}$ are the 0th- and 1st-order Bessel functions of the first kind. When a charged particle is generated at the cathode at $(z, t)=(0,0)$, the phase seen by it $\phi=\phi_{0}$ is the so-called injection phase of the particle. With an injection phase $\phi_{0}=180^{\circ}$, a negatively charged particle has its maximum energy gain after acceleration. The radial dependence of the rf field amplitudes is described by the Bessel functions. An increase of $r$ gives a rise of the transverse fields $E_{r}$ and $B_{\theta}$ but a reduction of the longitudinal field $E_{z}$. Figure 2(a) illustrates the rf fields seen by two electrons at positions of $r=0$ and $r=r_{0}$, respectively. The on-axis electron sees only the longitudinal field $E_{z}$ during acceleration, while the off-axis electron sees both a weaker longitudinal field $E_{z}$ and some transverse fields, $E_{r}$ and $B_{\theta}$, during acceleration. Since the amplitude of $E_{z}$ is radially dependent, the transit time of an electron depends on its radial position in the rf cavity. Even though an ideally deltafunction-like electron bunch could be emitted at the cathode with a planar pulse front extended in $r$ and propagate longitudinally in an rf accelerator, the shape of the electron-pulse front can be distorted into a curved surface by the

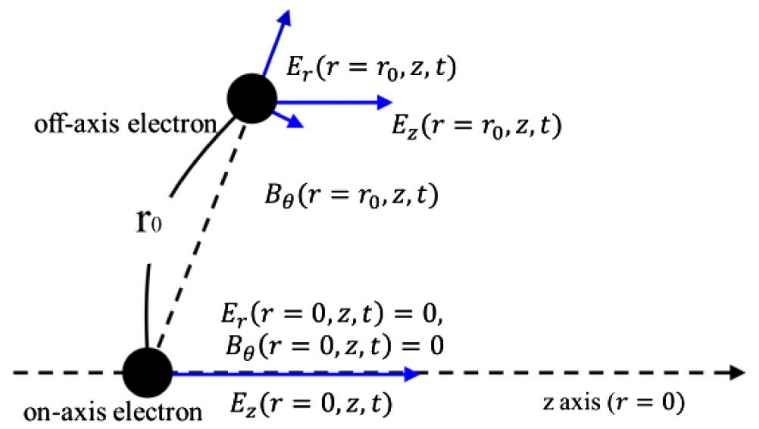

(a)

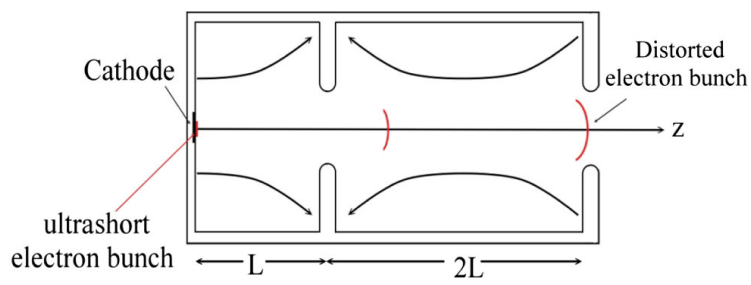

(b)

FIG. 2. (a) An on-axis electron sees only a longitudinal acceleration field, whereas an off-axis electron sees both longitudinal and transverse field components. (b) Simultaneously emitted electrons at the cathode plane cannot reach the accelerator exit at the same time due to the radial dependence of acceleration fields. radially dependent fields, as shown in Fig. 2(b). As a result, the simultaneously emitted electrons at the cathode plane may not reach the accelerator exit at the same time, and the longitudinal bunch length of the electrons is broadened. In the following, we consider this debunching effect in a BNL/SLAC/UCLA-type 1.6-cell, S-band rf photoinjector [22]. Table I lists the relevant parameters of the photoinjector under consideration. The total length of the photoinjector is $12.61 \mathrm{~cm}$, including a $1.79 \mathrm{~cm}$ long vacuum length after the acceleration cells.

For an electron accelerated by the radially dependent rf fields, the phase seen by the electron at $(z, t)$ is given by [21]

$$
\phi=\omega t-k_{0} z+\phi_{0}=\int_{0}^{z} \frac{\omega}{v_{z}} d z^{\prime}-k_{0} z+\phi_{0},
$$

where $v_{z}$ is the axial velocity of the electron, depending on $r, \phi$, and $z$. Comparing with an on-axis electron, the phase of an off-axis electron is retarded more during acceleration, since the off-axis electron propagates slower in the longitudinal direction. The phase spread $\Delta \phi$ between an electron at arbitrary $r$ and a reference particle at $r=0$ is defined as $\Delta \phi(r)=\phi(r)-\phi_{\text {ref }}(r=0)$.

From Eq. (7), the variation of $\phi$ along $z$ can be written as

$\frac{d \phi}{d z}=\frac{\omega}{v_{z}}-k_{0}=\frac{\omega}{c} \frac{\gamma}{\sqrt{\gamma^{2}-1}} \sqrt{1+\left(\frac{v_{r}}{v_{z}}\right)^{2}}-k_{0}$,

where $\gamma$ and $v_{r}$ are the Lorentz factor and the radial component of the electron velocity, respectively. From energy conservation, the variation of $\gamma$ along $z$ can be written as

$$
\frac{d \gamma}{d z}=\frac{e}{m_{0} c^{2}}\left(E_{z}+\frac{v_{r}}{v_{z}} E_{r}\right) \cong \frac{e}{m_{0} c^{2}} E_{z},
$$

where $e$ and $m_{0} c^{2}$ are the charge and rest energy of the electron, respectively. The approximation in Eq. (9) is valid, because $v_{r} \ll v_{z}$ and $E_{r} \ll E_{z}$ for a small $r$. From Eq. (9), it is evident that the energy of an on-axis electron is higher than that of an off-axis electron, as $E_{z}$ is reduced for an off-axis electron. From the Lorentz force equation, the

TABLE I. Parameters of the rf photoinjector.

\begin{tabular}{lc}
\hline \hline Parameter & Value \\
\hline rf resonance frequency $f_{\text {rf }}$ & $2.856(\mathrm{GHz})$ \\
Peak accelerating gradient $E_{0}$ & $80(\mathrm{MV} / \mathrm{m})$ \\
Cell length $L$ & $6.76(\mathrm{~cm})$ \\
Cathode position & $z=0(\mathrm{~cm})$ \\
Exit position (including a $1.79-\mathrm{cm}$ long & $z=12.61(\mathrm{~cm})$ \\
beam pipe) & Copper \\
Cathode material & \\
\hline \hline
\end{tabular}




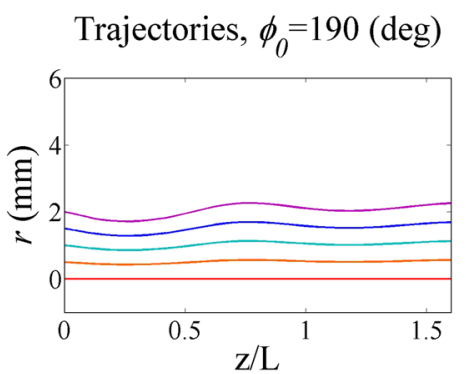

(a)
Trajectories, $\phi_{0}=250(\mathrm{deg})$

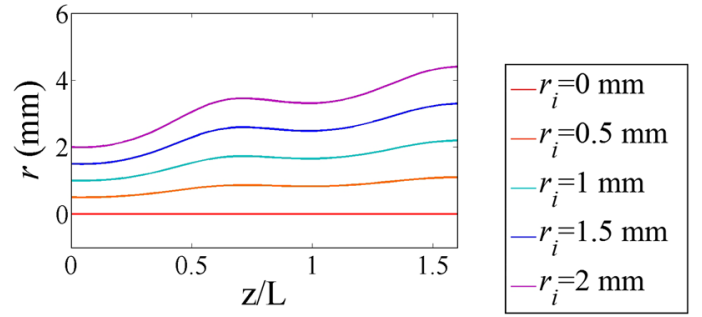

(b)

Accelerator exit, $\phi_{0}=190(\mathrm{deg}) \quad$ Accelerator exit, $\phi_{0}=250(\mathrm{deg})$

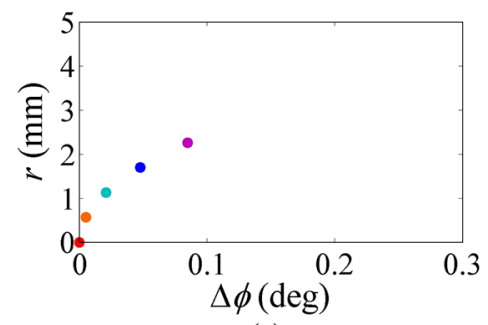

(c)

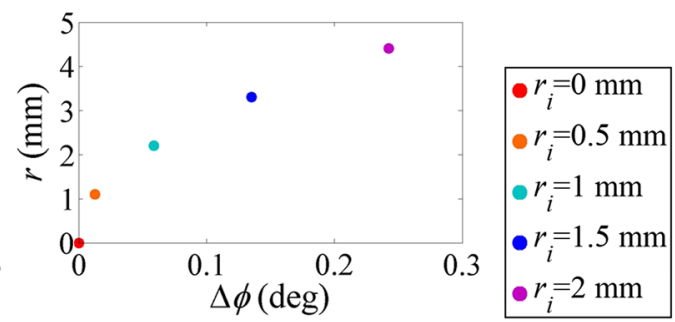

(d)

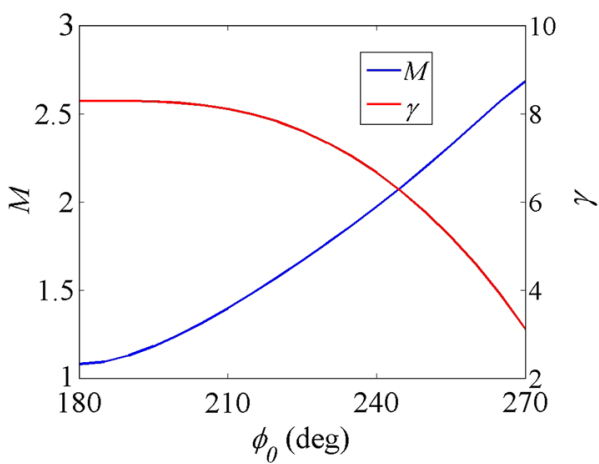

(e)

FIG. 3. Trajectories of electrons injected at $\phi_{0}=$ (a) $190^{\circ}$ and (b) $250^{\circ}$ in an rf injector. $\Delta \phi-r$ distributions of electrons with injection phase $\phi_{0}=$ (c) $190^{\circ}$ and (d) $250^{\circ}$ at the accelerator exit. (e) Radial magnification factor $M$ versus initial phase $\phi_{0}$ (blue curve), and Lorentz factor $\gamma$ versus $\phi_{0}$ (red curve). The $M-\phi_{0}$ and $\gamma-\phi_{0}$ relationships indicate an increased radial magnification and a decreased energy gain for off-crest acceleration, respectively.

variation of the radial velocity $v_{r}$ and the radial position $r$ of the electron along $z$ are, respectively,

$$
\frac{d v_{r}}{d z}=\frac{1}{\gamma m_{0}}\left[\frac{e}{v_{z}}\left(E_{r}-v_{z} B_{\theta}\right)-m_{0} v_{r} \frac{d \gamma}{d z}\right]
$$

and

$$
\frac{d r}{d z}=\frac{v_{r}}{v_{z}},
$$

with $v_{z}=\sqrt{\left(\gamma^{2}-1\right) c^{2}-\gamma^{2} v_{r}^{2}} / \gamma$. Since $E_{r}$ and $B_{\theta}$ vanish at $r=0, d v_{r} / d z$ and $d r / d z$ also vanish during acceleration for an on-axis electron. However, $d v_{r} / d z$ and $d r / d z$ keep changing during acceleration for an off-axis electron. Equations (8)-(11) form a set of coupled equations governing the dynamics of an electron motion in an accelerator. The phase spread of an electron bunch depends on how the electrons change their coordinates during acceleration with initial distributions in $\phi, \gamma, v_{r}$ and $r$ (denoted as $\phi_{0}, \gamma_{0}, v_{r 0}$, and $r_{i}$, respectively, for what follows).

\section{B. Evolution of an ultrashort electron bunch in the photoinjector}

Since the laser spot size on the cathode of a photoinjector is not infinitely small, the electrons are emitted from the cathode with a radial distribution. The relation between $r$ and $z$ or the trajectory of an electron under acceleration can be obtained by solving the coupled equations (8)-(11). Figures 3(a) and 3(b) show the trajectories of electrons emitted at different initial $r$ positions with injection phases $\phi_{0}=190^{\circ}$ and $250^{\circ}$, respectively. The on-axis electrons, as expected, remain propagating on the axis, whereas off-axis electrons oscillate along $r$ as they travel along $z$ due to the transverse fields. Comparing Fig. 3(a) with Fig. 3(b), one sees that for the radial displacement of the electrons 


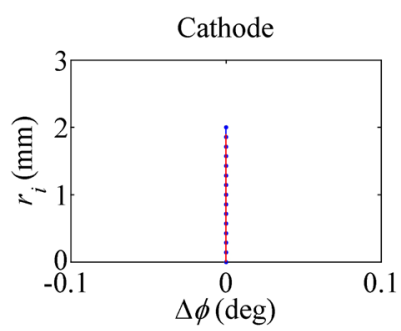

(a)

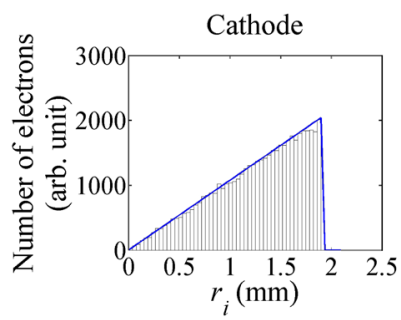

(d)

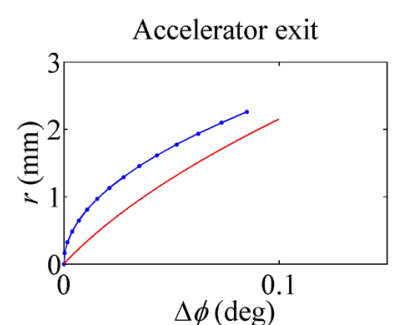

(b)

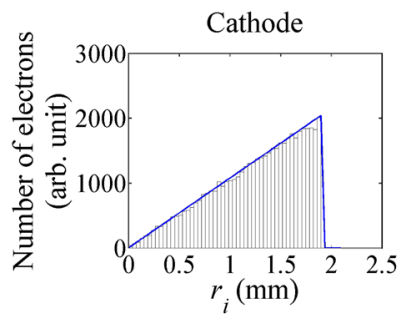

(e)

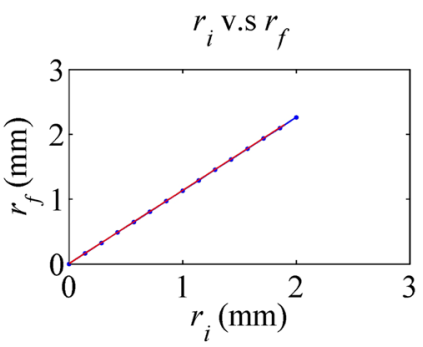

(c)

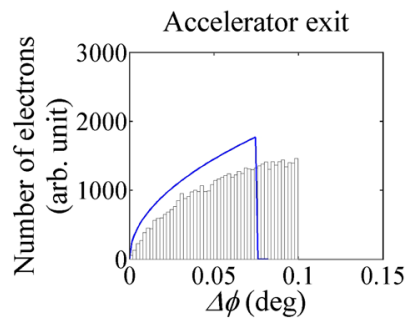

(f)

FIG. 4. (a)-(c) PARMELA simulation results (red lines) for a delta-function electron bunch before and after acceleration in the photoinjector, compared with our theoretical model (blue dotted lines). (a) $\Delta \phi-r$ distributions of an ultrashort electron bunch injected at $\phi_{0}=190^{\circ}$ at the cathode and (b) at the accelerator exit. (c) Linear relationship between $r_{i}$ and $r_{f}$ of the electrons. (d) Radial distributions of electrons at the cathode and (e) at the accelerator exit. (f) Longitudinal distribution of electrons at the accelerator exit. The radial and longitudinal distributions of an electron bunch can be regarded as the projections of the bunch onto the radial and longitudinal directions, respectively. The blue lines in (d)-(f) are calculated from the distribution functions (13)-(15), compared with the histograms of PARMELA simulation results.

injected at $\phi_{0}=250^{\circ}$ larger than that of $\phi_{0}=190^{\circ}$ such transverse motion of the off-axis electrons causes the electron to see an acceleration field $E_{z}(r)$ that is, in general, less than the axial acceleration field and fall behind the onaxis electron. Figures 3(c) and 3(d) show the corresponding distributions of the electrons in the $r$ and $\Delta \phi$ planes at the exit of the photoinjector, indicating the longitudinal spreading of electrons caused by the initial radial position $r_{i}$ and the injection phase $\phi_{0}$.

In the transverse direction, the oscillation amplitude of the electrons is also related to their initial radial position $r_{i}$ and injection phase $\phi_{0}$. The ratio $M \equiv d r_{f} / d r_{i}$ is defined as the magnification factor of an electron's radial coordinate at the accelerator exit, where $r_{f}$ denotes the final radial position of an electron. The blue curve in Fig. 3(e) summarizes the relationship between $M$ and $\phi_{0}$ for the photoinjector considered in this article, showing a less collimated output beam for a larger $\phi_{0}$. Also plotted in the same figure is the $\gamma-\phi_{0}$ curve of the accelerated electrons (red curve), indicating worsened collimation and reduced energy gain for an injection phase deviated away from $\phi_{0}=180^{\circ}$. As $\phi_{0}$ increases from $180^{\circ}$ to $270^{\circ}$, an off-axis electron sees an increased transverse field and moves further off axis.

The result calculated from the aforementioned simplified model is further compared with simulation using the particle tracking code PARMELA [14]. In our PARMELA simulation, the acceleration fields were numerically calculated by the electromagnetic-field code SUPERFISH [23] according to the design of the BNL/SLAC/UCLA S-band photoinjector [17]. The ultrashort electron bunch at the cathode is modeled as an infinitely thin particle layer with a zero bunch length in the longitudinal direction and a cutoff radius $\sigma_{c}=2 \mathrm{~mm}$ in the radial direction. In the simulation, a delta-function-like layer can be modeled by setting the injection phase of all particles to be the same in the particle distribution input file. The infinitely thin particle layer consists of uniformly distributed 50000 charged particles in the radial direction (flattop distribution). Such a deltafunction-like layer of electrons in the longitudinal direction allows us to exploit the impulse response of the accelerator system for the study of bunch distortion during particle acceleration. Neglecting the space charge effects, Figs. 4(a) and 4(b) show the $\Delta \phi-r$ distributions of the electrons injected at $\phi_{0}=190^{\circ}$ before and after acceleration, respectively. Both our simplified model and the PARMELA simulation result show that the initially upright $\Delta \phi-r$ distribution of electrons is bent gradually by the radially dependent rf fields during particle acceleration. The slight discrepancy between the two curves in Fig. 4(b) results from neglecting the high-order mode fields in the simplified model. In the paraxial region, $J_{1}\left(\eta_{0} r\right)=\eta_{0} r / 2-\left(\eta_{0} r\right)^{3} /$ $16+\left(\eta_{0} r\right)^{5} / 384 \approx \eta_{0} r / 2$, and the transverse fields $E_{r}$ and $B_{\theta}$ can be approximated as linear functions of $r$. Since the electron transit time through a photoinjector is approximately a constant for all electrons in a delta-function bunch, the transverse displacement of the electrons, proportional to twice the integrations of the transverse force over time, is therefore linearly proportional to $r$ in the paraxial limit. 
As a result, the relation between $r_{i}$ and $r_{f}$ of the electrons is approximately linear with a constant slope $M \equiv d r_{f} / d r_{i}$ in Fig. 4(c). The constant slope $M$ indicates that the transverse electron-beam size is broadened uniformly by $M$ times after acceleration.

Mathematically, the longitudinal and radial distribution functions of such an electron bunch at the cathode can be written as

$$
n(\phi)=N_{0} \delta\left(\phi-\phi_{0}\right)
$$

and

$$
n(r)=\int_{0}^{2 \pi} \frac{N_{0}}{\pi \sigma_{c}^{2}} \prod\left(r-\sigma_{c}\right) r d \theta=\frac{2 N_{0} r}{\sigma_{c}^{2}} \prod\left(r-\sigma_{c}\right),
$$

respectively, where $N_{0}$ is the total number of electrons and $\Pi(x)$ is a step function. $\Pi(x)=1$ for $x \leq 0$; otherwise, $\Pi(x)=0$. Figure 4(d) shows the radial distribution of electrons at the cathode, wherein the blue line calculated from Eq. (13) is overlapped with the particle's histogram obtained from the PARMELA simulations. Since $\sigma_{c}$ and $n(r)$ of the electron bunch are expected to be uniformly broadened in the $r$ direction by $M$ times in the paraxial region after acceleration, the radial distribution function of the electrons at the accelerator exit becomes

$$
n(r)=\frac{2 N_{0} r}{M^{2} \sigma_{c}^{2}} \prod\left(r-M \sigma_{c}\right),
$$

which, given $M$ from Fig. 4(c), is shown in Fig. 4(e) as a blue line outlining the particle's histogram at the accelerator exit. Moreover, the longitudinal distribution function of the accelerated electrons can be derived from $n(r)$ with a known $\Delta \phi-r$ relationship, $r=R(\Delta \phi)$ :

$$
n(\Delta \phi)=\frac{2 N_{0} R(\Delta \phi)}{M^{2} \sigma_{c}^{2}} \prod\left[R(\Delta \phi)-M \sigma_{c}\right] .
$$

Given the $\Delta \phi-r$ relationship in Fig. 4(b) and $n(r)$ in Fig. 4(e), the longitudinal profile of an ultrashort electron bunch changes from an initial delta function to a quartercycle sinelike function after acceleration, as shown by the blue line in Fig. 4(f); in other words, the longitudinal bunch length of the accelerated electrons is no longer infinitely small but broadened. The histogram in Fig. 4(f) is the result from the PARMELA simulation.

\section{Evolution of an ultrashort electron-pulse train in the photoinjector}

The previous example indicates that the longitudinal length of an ultrashort electron bunch is no longer infinitely small after the bunch is accelerated by the radially dependent fields in an rf accelerator. This result can be extrapolated to the acceleration of a train of ultrashort electron bunches. Figures 5(a)-5(c) show PARMELA simulations of an ultrashort electron-pulse train accelerated in the photoinjector, taking into account the space charge effects. In the simulation, the initial full length of the electron-pulse train is $1 \mathrm{ps}$, and the rf injection phase of the reference particle is chosen to be $\phi_{0}=190^{\circ}$ At the cathode the electrons are emitted at a pulse rate of $200 \mathrm{THz}$, and every electron microbunch has a flattop distribution in the transverse direction with an rms radius $\sigma_{r}=0.5 \mathrm{~mm}$, as shown in Fig. 5(a). The microbunches, as shown in Fig. 5(a), are generated by illuminating the cathode with a pulse-train laser. If the electron emission at the photocathode follows the driver laser pulse, the generation of a 200-THz electron-pulse train at the cathode would require a 200-THz pulse-train laser. Such a laser can be obtained from the beat note of harmonic laser fields. For example, it has been proposed that, to have a 282-THz laser-pulse train, one could in principle mix a gun driver laser at $266 \mathrm{~nm}$, the 4th harmonic of a Nd laser, with the first three harmonics of the laser [5]. In addition, overlapping a 200-THz laser

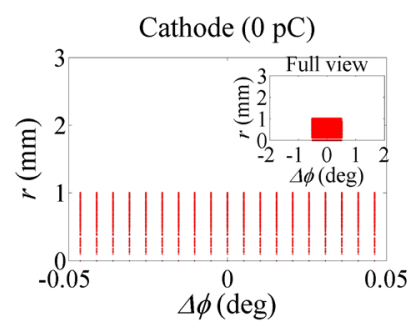

(a)

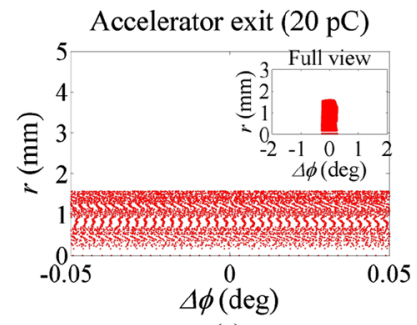

(c)

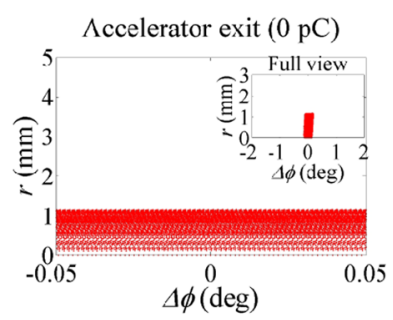

(b)

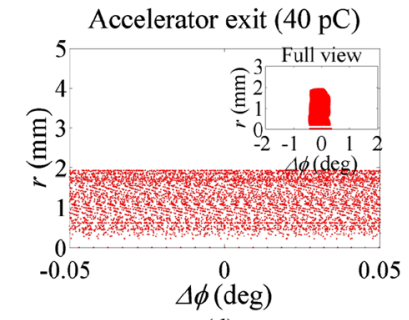

(d)

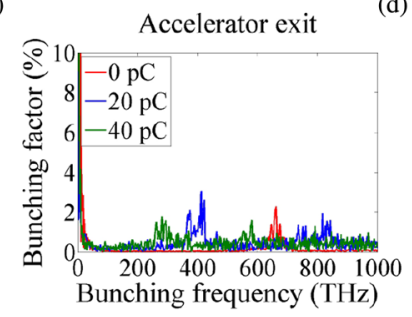

(e)

FIG. 5. (a) $\Delta \phi-r$ distribution of an electron-pulse train with an initial bunching frequency of $200 \mathrm{THz}$ at the cathode. $\Delta \phi-r$ distributions of the electron-pulse trains with total charges of (b) 0 (no space charge), (c) 20 , and (d) $40 \mathrm{pC}$ at the exit of the accelerator. (e) Bunching factor spectra of the output electronpulse trains with total charges of 0,20 , and $40 \mathrm{pC}$. The space charge field, in addition to the radially dependent acceleration fields, tends to smear out electron microbunches. Off-crest acceleration also introduces slight velocity bunching and shifts the bunching spectrum to higher frequencies. 
(wavelength $=1.5 \mu \mathrm{m}$ ) with an ultraviolet laser at $260 \mathrm{~nm}$ could gate the photoemission of electrons from a copper cathode at $200 \mathrm{THz}$ through the Schottky effect [24]. The polarization of the 200-THz laser is in the incident plane, so that the surface laser field enables the Schottky effect. The response time of the photoemission from a metal surface has been proved to be in the subfemtosecond region, derived from some recently developed attosecond resolved measurement techniques [25-28]. Figures 5(b)-5(d) show the distributions of the output electron-pulse trains with different total charges of 0,20 , and $40 \mathrm{pC}$, respectively. The zero charge case is equivalent to a simulation without the space charge effects. Figure 5(e) plots the bunching factor spectra of the output electron beams at the accelerator exit. It is seen from Fig. 5(b) that, with the radially dependent acceleration fields and without the space charge fields, the electron-pulse train is longitudinally compressed by about 3 times through velocity bunching with an injection phase around $\phi_{0}=190^{\circ}$, manifested by the increase of the bunching frequency shown in Fig. 5(e), where the peak bunching occurs at $670 \mathrm{THz}$. With both the space charge and the radially dependent acceleration fields, Figs. 5(c) and 5(d) show nonlinearly distorted electron microbunches at the accelerator exit. The repulsive space charge forces push electrons outward in both the longitudinal and transverse directions, make velocity bunching more difficult, and shift the output bunching factor spectra to lower frequencies.

\section{ELECTRON ACCELERATION WITH INJECTION PHASE COMPENSATION}

The previous studies indicate that, in the low-charge limit, the radial dependence of the rf field is the primary cause to the longitudinal debunching of ultrashort electrons during the acceleration process. To reduce the phase spread of an electron bunch at the exit of the accelerator, we can in principle tune the initial $\Delta \phi-r$ distribution of electrons at the cathode to compensate the longitudinal electron debunching. Specifically, the curved $\Delta \phi-r$ distribution of the electrons at the accelerator exit can be corrected by injecting an electron bunch with a properly countercurved $\Delta \phi-r$ distribution of electrons at the cathode.

To generate such phase-corrected electron bunches at the cathode, we propose a laser-pulse-front-shaping system for the driver laser, consisting of adaptive optical components such as lenses and deformable mirrors, to transform a planar laser-pulse front into a curved one [29-31]. This concept is illustrated in Fig. 6, where a laser-pulse-frontshaping system introduces a radially dependent optical group delay to an incident laser pulse to counterbalance the delayed transit time of the off-axis electrons in a short electron bunch. For example, if one adopts a piezoelectricdriven deformable mirror [31] for pulse-front shaping, a nanometer displacement on the mirror surface would give attosecond temporal resolution to the pulse-front shaping.

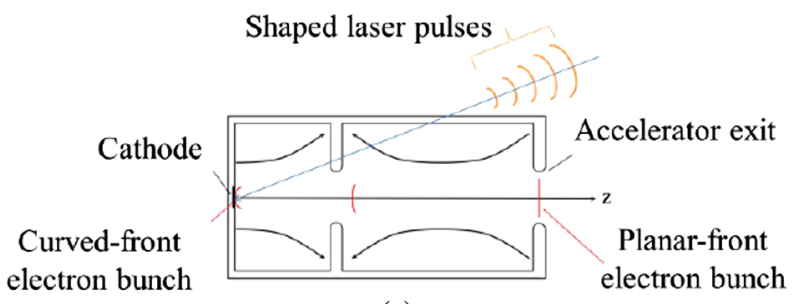

(a)

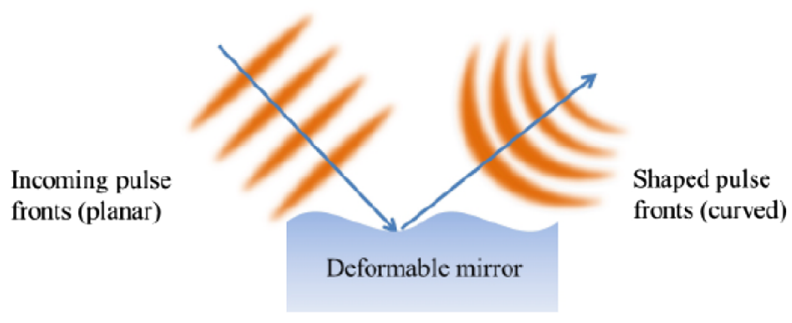

(b)

FIG. 6. (a) Illustration of the concept of a laser-pulse-frontshaping system, where adaptive optical components, such as deformable mirrors, can be used to tune the pulse front of an input laser pulse. (b) Illustration of the evolution of a curved-front electron bunch traveling through an rf accelerator.

Alternatively, one can propagate a laser pulse into a material and introduce a group delay to the pulse front. The group delay of a light wave in a glass material with a refractive index of 1.5 is about 1.67 fs per micrometer, which corresponds to an rf phase angle of $3 \times 10^{-5}{ }^{\circ}$ for an rf frequency of $2.856 \mathrm{GHz}$. The pulse-front-shaped laser, upon incident on a fast-response photocathode, is to induce photoemission of pulsed electrons with a replicated pulse front. We discuss in the following single- and multibunch acceleration of electrons with such an injection phase compensation (IPC) technique by using single and multiple curved-front laser pulses, respectively, to illuminate the cathode of a photoinjector.

\section{A. Single-bunch acceleration}

The amount of the phase distortion to an accelerated electron bunch depends on different conditions, such as the acceleration fields, the injection phase, the electron charge, the electron distribution, and so on. To cope with different acceleration conditions, we develop an iteration routine, as shown in Fig. 7, to numerically determine an optimal $\Delta \phi-r$ distribution of an electron bunch, so that the phase distortion to an accelerated electron bunch at the accelerator output is minimized.

The iteration routine is described as follows. Assume a delta-function electron bunch in the time domain containing initial $N$ electrons with their phase values described by $\Delta \hat{\phi}_{i}^{(0)}=\left(\Delta \phi_{i 1}^{(0)}, \Delta \phi_{i 2}^{(0)}, \ldots, \Delta \phi_{i N}^{(0)}\right)=\{\overrightarrow{0}\}$ at the cathode, and, after acceleration, a phase-distorted electron bunch described by $\Delta \hat{\phi}_{f}^{(0)}=\left(\Delta \phi_{f 1}^{(0)}, \Delta \phi_{f 2}^{(0)}, \ldots, \Delta \phi_{f N}^{(0)}\right) \neq\{\overrightarrow{0}\}$ at 


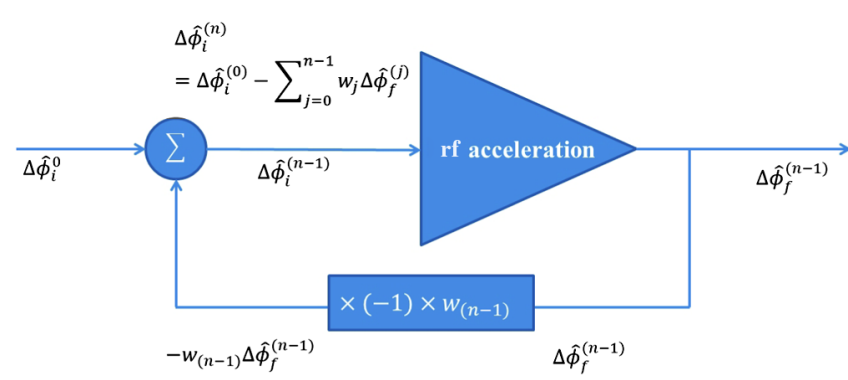

FIG. 7. An iteration loop to obtain the optimal injection phase for electrons at the cathode. The initial phase spread of electrons is iteratively modified by $n$ times to achieve an ideal final phase spread $\Delta \hat{\phi}_{f}^{(n)}=\{0\}$.

the accelerator exit, where the superscript (0) denotes the 0 th iteration, and the subscripts $i$ and $f$ denote the variables associated with the phase spreads before and after acceleration, respectively. In the PARMELA simulation, it is straightforward to track particles at different $r$ positions and extract their output phases after the acceleration. Comparison between the output and ideal phases offers a hint to compensate the injection phases of electrons for the next iterative calculation. In order to compensate the final phase spread, the initial phase values of electrons are iteratively corrected by $n$ times to $\Delta \hat{\phi}_{i}^{(n)}=\Delta \hat{\phi}_{i}^{(n-1)}-$ $\Delta \hat{\phi}_{f}^{(n-1)}=\Delta \hat{\phi}_{i}^{(0)}-\sum_{j=0}^{n-1} w_{j} \Delta \hat{\phi}_{f}^{(j)}$ until the final phase spread of the accelerated electrons is fully compensated, i.e., $\Delta \hat{\phi}_{f}^{(n)}=\{\overrightarrow{0}\}$, where $0<w_{j} \leq 1$ is a weighting factor chosen between iteration steps to avoid overcompensating the phase spread. For a highly charged electron bunch, the space charge effects often induce a large phase spread during acceleration. Hence, it is better to decrease the weighting factor and correct the initial distribution carefully to avoid getting a divergent result. At the beginning, the iteration routine usually starts with weighting factors $w_{j}=1$ for $0 \leq j \leq n-1$. If the phase spread of the electron bunch becomes larger after each iterative loop, it means that the phase spread has been overcompensated, and a smaller weighting factor is used in the next iterative loop.

Figure 8 is a result of our simulation study to obtain a delta-function electron bunch at the accelerator exit for an initial flattop distribution of electrons on the cathode plane $\left(\sigma_{r}=0.5 \mathrm{~mm}\right.$ at the cathode). Figures 8(a)-8(c) show the required initial distributions of electrons at the cathode for total bunch charges of 0,20 , and $40 \mathrm{pC}$, respectively, to achieve the corresponding delta-function distributions of electrons in Figs. 8(d)-8(f) at the exit of the accelerator. For comparison, we also plot in Fig. 9 the same simulation study, except that the initial distribution of the cathode electrons is Gaussian with $\sigma_{r}=0.5 \mathrm{~mm}$ in the radial direction. Without space charge effects, the optimal initial electron distributions in Figs. 8(a) and 9(a) appear the same, regardless of different transverse distributions of the cathode electrons. This is understood by noting that the required amount of IPC at the cathode depends only on the radial position of the electron. With space charge effects, the required injection phase compensations shown in Figs. 8(b), 8(c), 9(b), and 9(c) become more sophisticated. In particular, those off-axis electrons with large $r$ in a Gaussian bunch experience both relatively large nonlinear acceleration and space charge fields, resulting in highly nonlinear dependence of the compensating phase on $r$.

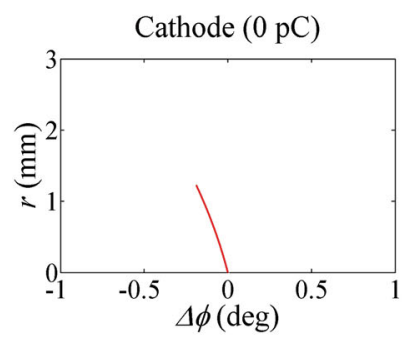

(a)

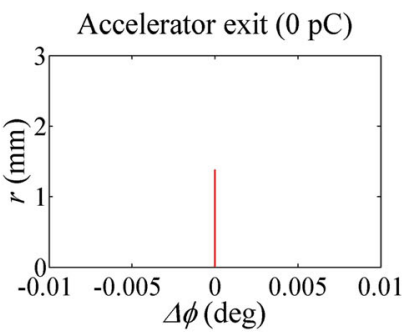

(d)

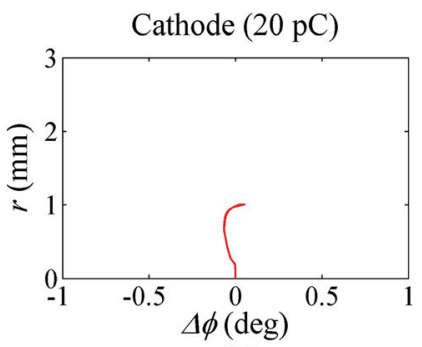

(b)

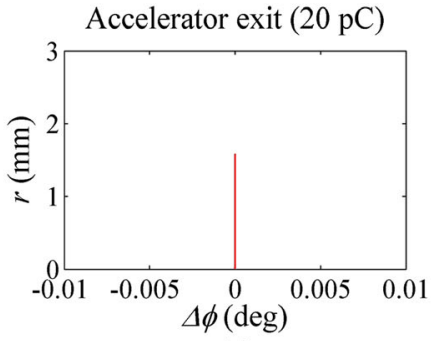

(e)

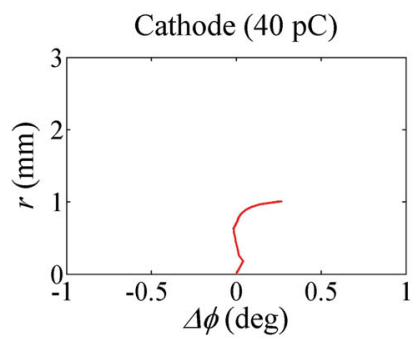

(c)

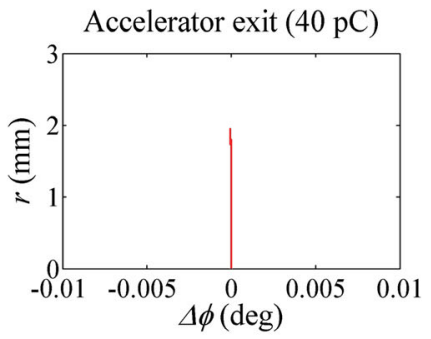

(f)

FIG. 8. $\Delta \phi-r$ distributions of electron bunches with flattop transverse profiles at the cathode for total charges of 0,20 , and 40 pC (a)-(c) before and (d)-(f) after acceleration, respectively. The injection phase of the reference particle for this simulation study is $\phi_{0}=190^{\circ}$. With IPC, the distribution of the electron bunch at the exit of the accelerator is fully restored to a delta function. 


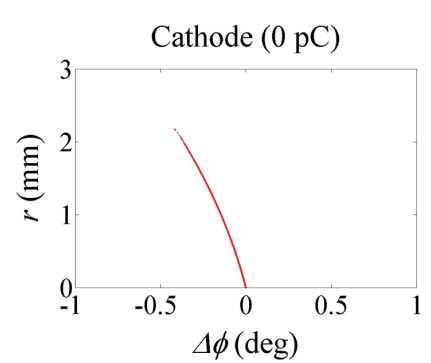

(a)

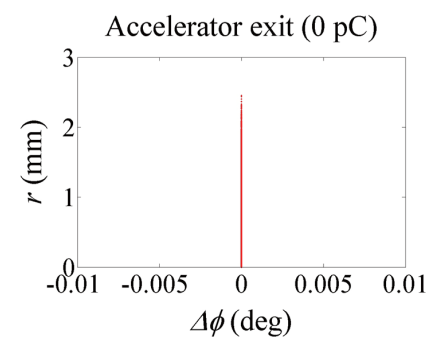

(d)

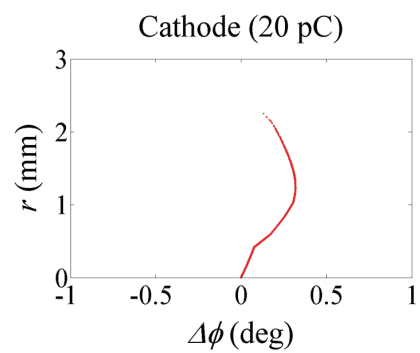

(b)

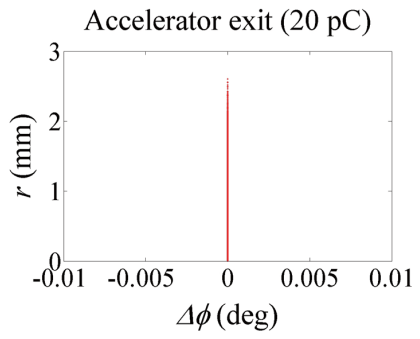

(e)

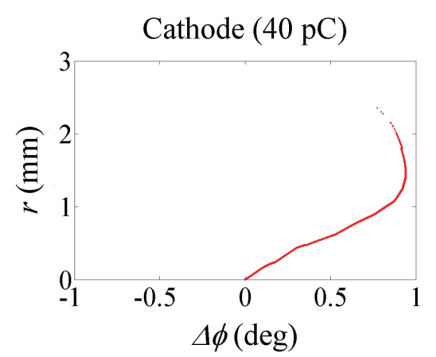

(c)

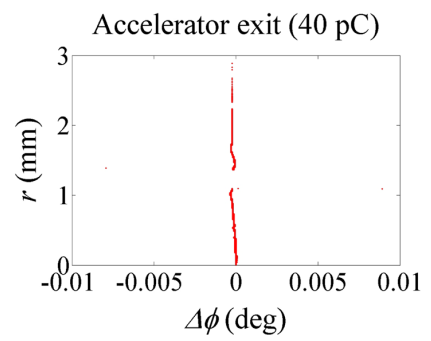

(f)

FIG. 9. Studies similar to Fig. 8, except that the initial transverse electron distribution at the cathode is Gaussian with an rms radius of $0.5 \mathrm{~mm}$. With the space charge effects, the amount of required IPC is larger and more nonlinear, compared with that for a transversely flattop beam in Fig. 8 .

Despite IPC, the phase spread of electrons cannot be fully compensated in Figs. 8(f) and 9(f), and furthermore a transverse beam breakup is observed in Fig. 9(f). Since the broken beam still has a delta-function-like profile in the longitudinal direction, it is unlikely to affect the overall spontaneous radiation power. However, the hole in the beam is to influence the spatial coherence of the electron radiation and also implies inefficient interactions between electrons and radiation during stimulated radiation. For a single electron bunch with $\sigma_{r}=0.5 \mathrm{~mm}$, the simulation results indicate that the highest charge that IPC can fully compensate is smaller than $40 \mathrm{pC}$.

In the phase compensation algorithm, an infinitely thin layer represents the pulse front of the electrons. For electron bunches with the same bunch profile but different bunch lengths, the curvature of their $\Delta \phi-r$ distributions calculated by IPC would be the same. Therefore, a properly shaped pulse front can correct not only an infinitely thin bunch, but also longitudinally thicker ones with the same bunch profile. For example, the shaped pulse fronts for correcting two equally charged electron bunches with bunch lengths of 1 and $10 \mathrm{fs}$ are the same. Because a thinner bunch is more like the infinitely thin particle layer, IPC compensates a thinner bunch better than a thicker one. On the other hand, when the driver laser-pulse duration is much larger than the longitudinal bunch broadening resulting from the radially dependent fields during acceleration, the improvement of IPC becomes negligible. The phase spread of single electron bunches at the accelerator exit is usually smaller than $1^{\circ}$, as shown in Figs. 3(c), 3(d), and 4(b), corresponds to a temporal length about 1 ps. As a result, IPC is worth being used for compensating electron bunches with initial bunch length not much longer than 1 ps.

\section{B. Partially phase compensated multibunch acceleration}

In practice, it is easier to illuminate the photocathode with a fast laser-pulse train containing identical pulses to generate identical electron microbunches at the cathode. However, identical electron bunches emitted at different times at the cathode are accelerated at different rf phases, having different phase distortions at the accelerator exit. We present in this section a technique of partial phase compensation (PPC) for multibunch acceleration, in which only the initial phase spread of the central microbunch is modified iteratively through the routine depicted in Fig. 7 to achieve a delta-function-like electron bunch at the accelerator exit. All the rest of the electron bunches are generated at the cathode by the driver laser pulses optimized for the central bunch. Figures 10(a)-10(c) show the initial particle distributions of such partially phase compensated electron-pulse trains containing total charges of 0 , 20 , and $40 \mathrm{pC}$, respectively. In the longitudinal direction, the electron microbunches repeat at $200 \mathrm{THz}$ in a 1-ps square envelope. In the transverse direction, the electron beam has a flattop distribution with an rms bunch radius of $0.5 \mathrm{~mm}$ at the cathode. Shown in Figs. 10(d)-10(f) are the particle distributions at the exit of the accelerator for the input conditions in Figs. 10(a)-10(c), respectively. It is seen that each output beam successfully generates a deltafunction bunch at the pulse center from the iteration routine but contains some distorted bunches accelerated at different rf phases. The driver laser pulse, which fully compensates 


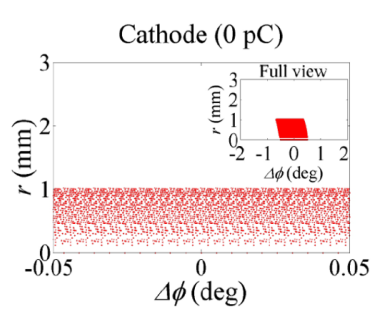

(a)

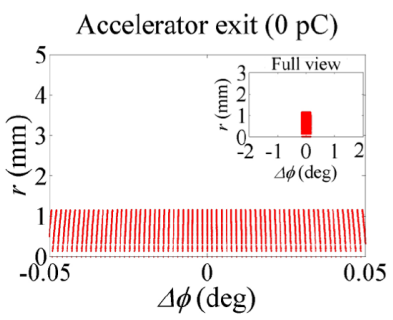

(d)

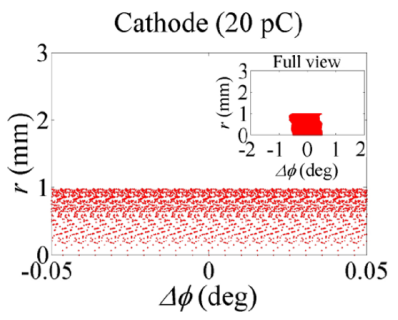

(b)

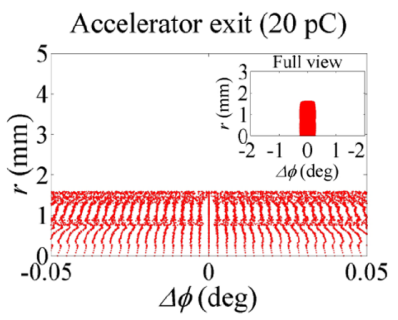

(e)

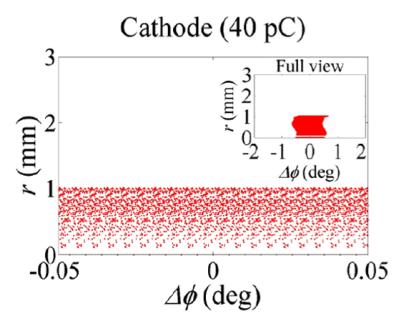

(c)

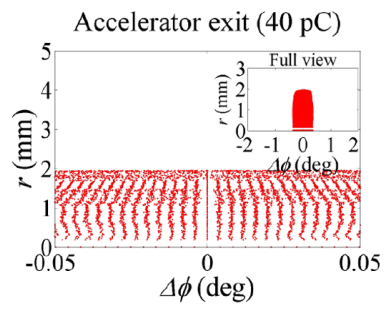

(f)

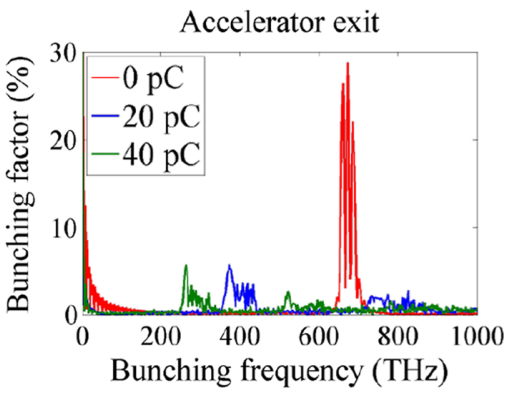

(g)

FIG. 10. (a)-(c) Initial $\Delta \phi-r$ distributions of particles for the partially phase compensated electron-pulse trains containing total charges of 0,20 , and $40 \mathrm{pC}$ at the cathode, respectively. (d)-(f) $\Delta \phi-r$ distributions of the accelerated electron-pulse trains at the exit of the accelerator with inputs in (a)-(c), respectively. (a)-(c) are generated through photoemission of electrons from a train of identical driver laser pulses incident on the cathode with the pulse front of the central laser pulse optimized to generate a delta-function bunch at the pulse-train center after acceleration. (g) Bunching spectra of the partially phase compensated electron-pulse trains at the exit of the accelerator, indicating deterioration of electron bunches from the acceleration and space charge fields.

the phase spread of the central microbunch, can only partially compensate that of off-center microbunches at the accelerator exit. Figure 10(g) summarizes the bunching spectra calculated from the partially compensated electronpulse trains in Figs. 10(d)-10(f), indicating deteriorated bunches from acceleration and space charge fields, despite optimization of the injection phase of the electron bunch at the center of the macropulse.

Comparison between Figs. 5(e) and 10(g) reveals that the PPC has effectively improved the bunching quality of the electron-pulse train after acceleration. Taking the $0-\mathrm{pC}$ electron beam as an example, without any phase compensation, the bunching factor at the accelerator exit is smaller than $2 \%$ at $f_{b} \sim 670 \mathrm{THz}$ in Fig. 5(e), whereas, with the PPC, the bunching factor increases to about $30 \%$ at the same bunching frequency as shown in Fig. 10(g). According to the relationship between the bunching factor and the radiation power [1-3], the radiation power of the electron-pulse train in an undulator could be enhanced by about 225 times by the PPC technique. As the total charge and pulse rate increase, compensating the phase spread to obtain a highly bunched beam becomes more difficult. Nevertheless, with PPC, the peak bunching factor for beams with total charges of 20 and $40 \mathrm{pC}$ is still enhanced by a few percent.

\section{Fully phase compensated multibunch acceleration}

The PPC technique only corrects the emission phase of the central electron bunch in a bunch train to generate a delta-function bunch at the exit of the accelerator. When the space charge effects take place in a high density beam, the same laser pulse optimized for the emission of the central bunch cannot always compensate the worsened phase spread of some marginal bunches. Assuming the pulse front of individual laser pulses can be tailor designed and implemented, we study in this section a full phase compensation (FPC) technique to generate a train of deltafunction electron bunches at the exit of the photoinjector. 


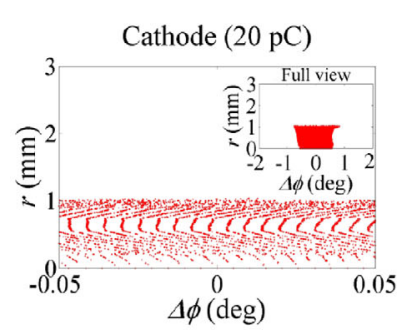

(a)

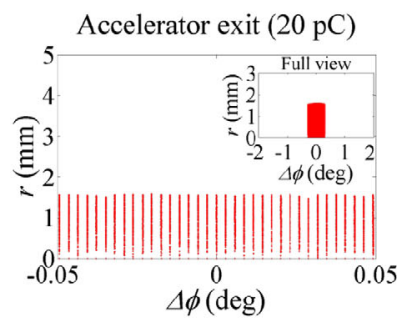

(d)

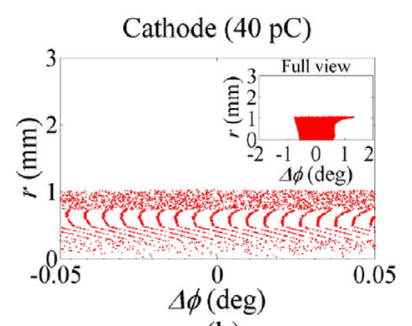

(b)

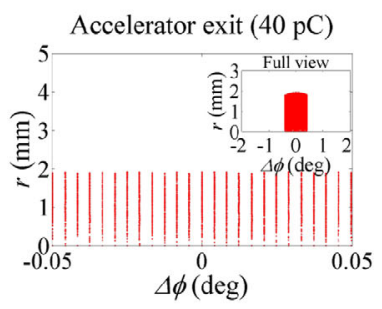

(e)

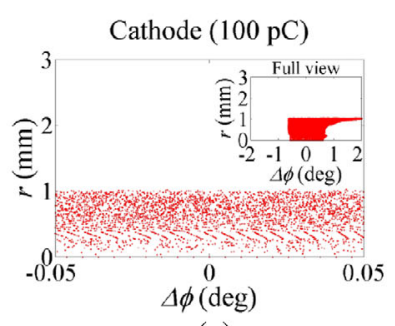

(c)

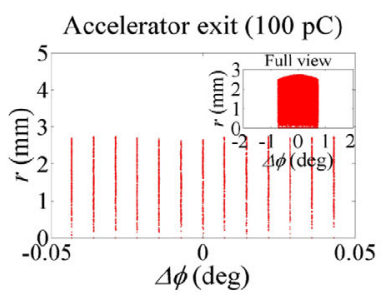

(f)

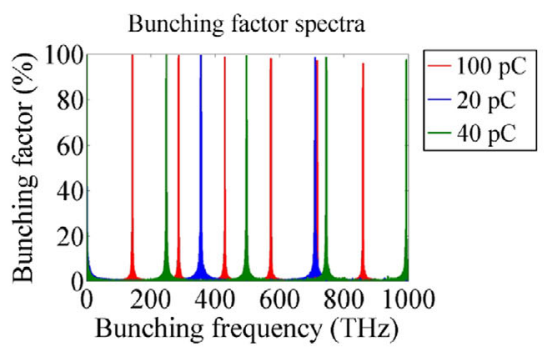

(g)

FIG. 11. (a)-(c) Initial $\Delta \phi-r$ distributions of particles for the fully phase compensated electron-pulse trains containing total charges of 20,40, and $100 \mathrm{pC}$ at the cathode, respectively. (d)-(f) $\Delta \phi-r$ distributions of the accelerated electron-pulse trains at the exit of the accelerator with inputs in (a)-(c), respectively. (a)-(c) are generated through photoemissions of electrons from a train of individually pulse-front-optimized driver laser pulses incident on the photocathode. The pulse rate at the cathode in this simulation is $200 \mathrm{THz}$. (g) Bunching spectra of the output electron-pulse trains with FPC, indicating nearly perfect bunching regardless of the bunch charges.

In our PARMELA simulation, we optimized the initial phase spread of each microbunch with the aforementioned iterative routine until the phase spreads of all the microbunches are fully compensated after acceleration. Figures 11(a)-11(c) show the optimized particle distributions of 200-THz electron-pulse trains at the cathode for 20, 40, and $100 \mathrm{pC}$ charges, respectively. Figures 11(d)-11(f) show the particle distributions at the exit of the accelerator corresponding to the input conditions in Figs. 11(a)-11(c), respectively. Comparing the FPC result in Figs. 11(d) and 11(e) with the PPC result in Figs. 10(d) and 10(e) of the same charges, we find that all the individually phase-corrected electron microbunches in Figs. 11(d) and 11(e) retain a delta-function bunch shape after acceleration. We further increase the total charge of the electron-pulse train to $100 \mathrm{pC}$ in the simulation, and the result in Fig. 11(f) indicates that the phase spread of each electron microbunch is still fully compensated for this high density beam. This FPC technique is apparently more effective than the PPC technique in compensating all the phase spreads in an electron-pulse train under nonlinear acceleration and space charge fields. Quantitatively, Fig. 11(g) shows nearly $100 \%$ bunching at the exit of the accelerator for all three cases with FPC. Such a perfectly bunched electron-pulse train does not have to undergo the selfamplified spontaneous emission (SASE) process in the undulator to further increase its bunching factor and radiation power, whereas it radiates coherent and highpower electromagnetic waves while entering the undulator. Similar to the previous results in Fig. 10, as the total beam charge increases, the bunch radius increases during acceleration and the output bunching spectrum is shifted toward a lower frequency. This simulation study shows that the FPC technique is flexible and effective to correct the phase spreads of ultrashort electron bunches during rf acceleration. This technique relies on the generation of ultrashort laser pulses with individually tailor-designed pulse fronts from the driver laser of a photoinjector. Whether this laser technique could be available now or in the future is beyond the scope of the discussion in this paper. 


\section{CONCLUSIONS}

We have studied the longitudinal phase spread of delta-function electron bunches during acceleration in a photoinjector and successfully corrected the spread by using laser pulses with tailor-designed pulse fronts at the photocathode.

For a low energy electron bunch, the distribution of electrons is susceptible to both the acceleration field and the space charge field. In the low-charge limit, both our theoretical model and PARMELA simulation indicate that the electrons are debunched in both the longitudinal and transverse directions by the spatially nonuniform acceleration fields. As the bunch charge increases, our computer simulation shows that the space charge field further worsens the bunching quality of the electrons during acceleration. For example, a 40-pC initially perfectly bunched electron-pulse train at $200 \mathrm{THz}$ at the photocathode is deteriorated to have a peak bunching factor of $2 \%$ at $280 \mathrm{THz}$ after acceleration in the S-band photoinjector.

To preserve an ultrashort electron bunch, we propose the IPC technique applied to the electrons at the emission by illuminating the photocathode with a pulse-front optimized laser pulse. The laser-pulse front is initially designed through a simulation-iteration routine and implemented through an adaptive optical system. For a single electron bunch with a total charge up to $40 \mathrm{pC}$, our simulation study shows that the phase spread of the electrons is well compensated after acceleration with the IPC technique.

For an electron-pulse train, different microbunches accelerated at different rf phases experience slightly different amounts of phase distortion. Applying the IPC technique to the central microbunch of an electron-pulse train, we can retain significant value of the bunching factor after acceleration, although in this technique the phase spread of the marginal electron bunches is just partially compensated. In our simulation study, such a PPC technique increases the $2 \%$ peak bunching factor for the case without PPC to about $6 \%$ for a $40-\mathrm{pC}, 200-\mathrm{THz}$ beam. According to the square proportional relationship between the bunching factor and the radiation power, the power of undulation radiation is expected to be increased by about 9 times with PPC.

To provide IPC to all the electron microbunches in a pulse train, we further assume a laser technology capable of providing a laser-pulse train with each laser-pulse front individually optimized to induce a phase-corrected electron bunch at the photocathode. With such a FPC technique, we confirmed from our simulation study that the phase spread of every electron microbunch in a $200-\mathrm{THz}$ pulse train can be nicely compensated at the accelerator exit. The simulation result shows nearly a $100 \%$ bunched electron-pulse train with a total charge up to $100 \mathrm{pC}$ at the exit of a photoinjector. In a SASE FEL, the bunching factor of the electron beam usually achieves a high value after a longdistance propagation in the undulator. Sending an $\sim 100 \%$ bunched electron-pulse train into the undulator is able to get highly coherent radiations directly. Thus, the required length of the undulator could be substantially reduced.

In our simulation-iteration routine, we set a goal to generate delta-function electron bunches at the exit of the photoinjector. In practice, the output point can be at any location of interest, such as the entrance or exit of a downstream high energy linac. Once the electron bunch reaches high energy, phase spread from the space charge effects is less of a concern. Moreover, it is convenient to generate ultrashort electron bunches with IPC at the location of the sample in the applications of UED or UEM.

A laser-shaping technology to meet the requirements for the proposed short-bunch photoinjector is also a topic that deserves further investigation. With adaptive optics, the technology is ready for generating a single pulse-frontshaped ultrashort laser pulse. To produce an ultrashort electron bunch at the cathode, a photocathode with ultrashort response time is needed. Some experiments have successfully demonstrated that the response time of photoemission from metal surfaces is in the subfemtosecond region. The simulation results for single- and multibunch cases show that the IPC scheme is suitable for correcting electron bunches with a bunching frequency ranging from 0 to $200 \mathrm{THz}$. To generate a 200-THz laser-pulse train with pulse-front correction to one or many pulses, it is probably feasible to copropagate a UV laser beam at $260 \mathrm{~nm}$ and a properly phase-modulated laser beam at $1.5 \mu \mathrm{m}$ to a copper cathode of a photoinjector. However, the details of the laser technology still need to be investigated.

\section{ACKNOWLEDGMENTS}

This work is supported by the Ministry of Science and Technology under Contracts No. NSC 99-2112-M-007013-MY3 and No. NSC 102-2112-M-007-002-MY3. F. H.C. acknowledges Ph.D. scholarship support from National Synchrotron Radiation Research Center.

[1] A. Gover, Phys. Rev. ST Accel. Beams 8, 030701 (2005).

[2] Y. C. Huang, Appl. Phys. Lett. 96, 231503 (2010).

[3] J. G. Neumann, P. G. O'Shea, D. Demske, W. S. Graves, B. Sheehy, H. Loos, and G. L. Carr, Nucl. Instrum. Methods Phys. Res., Sect. A 507, 498 (2003).

[4] F. H. Chao, C. H. Chen, Y. C. Huang, and P. J. Chou, in Proceedings of the 4th International Particle Accelerator Conference, IPAC-2013, Shanghai, China, 2013, edited by N. Zhao (JACoW, Shanghai, China, 2013), pp. 3109-3111.

[5] F. H. Chao, C. H. Chen, K. Y. Huang, and Y. C. Huang, in Proceedings of the 33rd Free Electron Laser Conference, Shanhai, 2011 (JACoW, Shanghai, 2011), pp. 3109-3111.

[6] M. Boscolo, M. Ferrario, I. Boscolo, F. Castelli, and S. Cialdi, Nucl. Instrum. Methods Phys. Res., Sect. A 577, 409 (2007).

[7] R. K. Li and C. X. Tang, Nucl. Instrum. Methods Phys. Res., Sect. A 605, 243 (2009). 
[8] R. K. Li, C. X. Tang, Y. Du, W. Huang, Q. Du, J. Shi, L. Yan, and X. Wang, Rev. Sci. Instrum. 80, 083303 (2009).

[9] J. B. Hastings, F. M. Rudakov, D. H. Dowell, J. F. Schmerge, J. M. Castro, S. M. Gierman, H. Loos, and P. M. Weber, Appl. Phys. Lett. 89, 184109 (2006).

[10] P. Musumeci, J. T. Moody, and C. M. Scoby, Ultramicroscopy 108, 1450 (2008).

[11] Y. Murooka, N. Naruse, S. Sakakihara, M. Ishimaru, J. Yang, and K. Tanimura, Appl. Phys. Lett. 98, 251903 (2011).

[12] J. Yang, K. Kan, T. Kondoh, Y. Yoshida, K. Tanimura, and J. Urakawa, Nucl. Instrum. Methods Phys. Res., Sect. A 637, S24 (2011).

[13] J. Yang, N. Naruse, K. Kan, T. Kondoh, Y. Yoshida, and K. Tanimura, in Proceedings of the 3rd International Particle Accelerator Conference, IPAC-2012, New Orleans, LA, 2012 (JACoW, New Orleans, LA, 2012), pp. 4170-4174.

[14] L. M. Young, Los Alamos National Laboratory Report No. LA-UR-96-1835, revised April 22, 2003.

[15] M. J. de Loos, S. B. van der Geer, Y. M. Saveliev, V. M. Pavlov, A. J. W. Reitsma, S. M. Wiggins, J. Rodier, T. Garvey, and D. A. Jaroszynski, Phys. Rev. ST Accel. Beams 9, 084201 (2006).

[16] M. Khojoyan, M. Krasilnikov, and F. Stephan, in Proceedings of the 5th International Particle Accelerator Conference, IPAC-2014, Dresden, 2014 (JACoW, Dresden, 2011), pp. 2958-2961.

[17] C. Limborg-Deprey, SLAC, 2275 Sand Hill Road, MS18, Menlo Park, USA, Report No. SLAC-PUB-11604.

[18] I. V. Bazarov, D. G. Ouzounov, B. M. Dunham, S. A. Belomestnykh, Y. Li, X. Liu, R. E. Meller, J. Sikora, and C. K. Sinclair, Phys. Rev. ST Accel. Beams 11, 040702 (2008).
[19] G. Penco, M. Danailov, A. Demidovich, E. Allaria, G. De Ninno, S. Di Mitri, W. M. Fawley, E. Ferrari, L. Giannessi, and M. Trovó, Phys. Rev. Lett. 112, 044801 (2014).

[20] L. Serafini, Nucl. Instrum. Methods Phys. Res., Sect. A 340, 40 (1994).

[21] K. J. Kim, Nucl. Instrum. Methods Phys. Res., Sect. A 275, 201 (1989).

[22] D. T. Palmer, X. J. Wang, R. H. Miller, M. Babzien, I. Ben-Zvi, C. Pellegrini, J. Sheehan, J. Skaritka, H. Winick, M. Woodle, and V. Yakimenko, in Proceedings of the Particle Accelerator Conference, Vancouver, BC, Canada, 1997 (JACow, New York, 1997), p. 2687.

[23] J. H. Billen and L. M. Young, Los Alamos National Laboratory Report No. LA-UR-96-1834, revised February 6, 2003

[24] H. Tomizawa, H. Dewa, A. Mizuno, T. Taniuchi, and H. Hanaki, in Proceedings of the 32nd Free Electron Laser Conference, Malmö, Sweden (JACow, Sweden, 2010), pp. 640-643.

[25] A. L. Cavalieri et al., Nature (London) 449, 1029 (2007).

[26] P. Hommelhoff, C. Kealhofer, and M. A. Kasevich, Phys. Rev. Lett. 97, 247402 (2006).

[27] S. Neppl, Physik Department E20, Technische Universitat Munchen (TUM), report, 2012.

[28] E. Goulielmakis, V. S. Yakovlev, A. L. Cavalieri, M. Uiberacker, V. Pervak, A. Apolonski, R. Kienberger, U. Kleineberg, and F. Krausz, Science 317, 769 (2007).

[29] M. Kempe and W. Rudolph, Phys. Rev. A 48, 4721 (1993).

[30] H. Nosatot, T. Itatanit, M. Murakawat, T. Higuchit, and $\mathrm{H}$. Noguchi, IEEE International Conference on Systems, Man, and Cybernetics, IEEE SMC-2014, San Diego, 2004 (IEEE, San Diego, CA, 2014), pp. 3675-3679).

[31] P. Y. Madec, in Proceedings of SPIE 2012, San Diego, 2012 (SPIE, San Diego, CA, 2014), Vol. 8447, pp. 8447051-844705-18. 